\title{
Cloud monitoring system by Visible-light Fisheye CCD
}

\section{Takayuki Tomida ${ }^{* \dagger}$}

Shinshu University, Nagano, Nagano, Japan

E-mail: tomidadcs.shinshu-u.ac.jp

\section{Yasunori Saito}

Shinshu University, Nagano, Nagano, Japan

E-mail: saitohdcs.shinshu-u.ac.jp

\section{Ryo Nakamura}

Shinshu University, Nagano, Nagano, Japan

E-mail: 17w2064adshinshu-u.ac.70

\section{Katsuya Yamazaki}

Earthquake Research Institute, The University of Tokyo, Bunkyo, Tokyo, Japan

E-mail: kvamazakideri.u-tokvo.ac.jp

\section{for the TA Collaboration}

Telescope Array (TA) is an international joint experiment observing ultra-high energy cosmic rays. TA employs the fluorescence detection technique to observe cosmic rays. In this technique, the existence of cloud significantly affects the quality of data. Therefore, cloud monitoring provides important information.

We are developing methods for evaluating night sky weather with pictures taken by a chargecoupled device (CCD) camera. We found that there was a change in the brightness of the whole image due to the weather. We tried to judge the weather only by the brightness of the whole image and gained only limited success. On the other hand, it is expected that there will be a correlation between the amount of clouds and the number of stars appearing in the image. We first devised a method to count the number of stars in the image. The stars are clusters of very bright pixels in the image, the method developed focusing on this has worked well.

Currently, we are developing evaluation methods to compare detected stars and catalogs.

35th International Cosmic Ray Conference - ICRC2017

10-20 July, 2017

Bexco, Busan, Korea

\footnotetext{
*Speaker.

${ }^{\dagger}$ Takayuki Tomida
} 


\section{Introduction}

Telescope Array (TA) experiment has observed Ultra High Energy Cosmic Rays (UHECRs) with energy above $10^{18} \mathrm{eV}$ using a hybrid technique of air fluorescence detection and particle counting in Utah, U.S [W]. In the fluorescence technique, we observe the fluorescence emission of nitrogen in the atmosphere excited by cosmic-ray secondary particles using fluorescence detectors (FDs). FDs are located in 3 stations (BR, LR and MD), with each station having $12 \sim 14$ telescopes. The distance between detectors and emitting locations of fluorescence light is several tens $\mathrm{km}$. BR and LR FD-stations are operated remotely from Delta, located $30 \mathrm{~km}$ from BR FDs. Therefore, we need to carefully monitor atmospheric parameters, select observation nights for analyzing data when using this technique. TA experiment has three night-sky weather cameras to monitor the environment (sky and weather) around FDs. The monitors have been located at BR, LR and Central Laser Facility (CLF) sites since August 2010. The location map of TA's detectors and facilities is shown in Fig. $\square$. The night-sky weather monitor takes night-sky images every minute in stable operation. These images are used by FD operators for judgment on the environment for observation and the possibility of operation. On another hand, an "Eye-scan code" recorded by the FD operators with traditional method is used for data selection to take into account the weather of observation nights. The Eye-scan code is recorded at the MD FD-station, at 30 minutes to 1 hour intervals, reliably. In this paper, we discuss methods of quantifying the amount of cloud in night sky using images of the night-sky weather monitor.

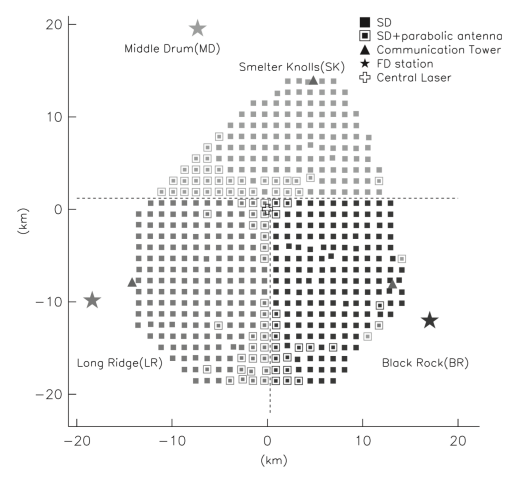

Figure 1: Detectors and facilities location in TA site. tem.

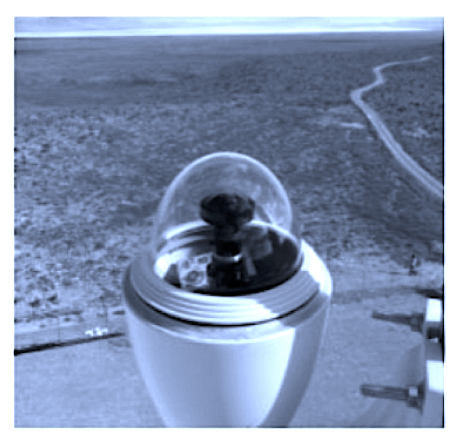

Figure 2: Image of Night sky weather monitor sys-
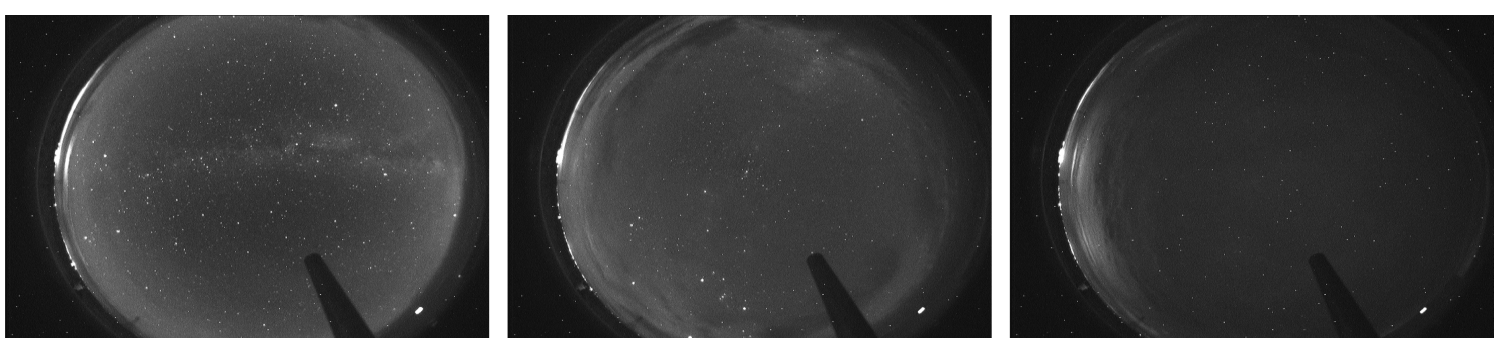

Figure 3: Example of night sky image by our system in 2014/Nov./20. Left: Clear weather at 02:09(UTC), Center: Partly overcast at 06:23(UTC), Right: Overcast at 11:00(UTC) 


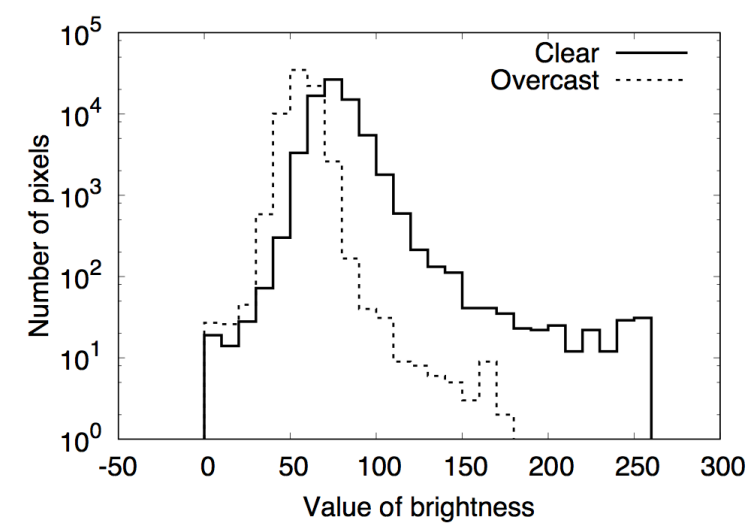

Figure 4: Distribution of brightness value of clear sky and cloudy sky.

\section{Night sky weather monitor system}

Night-sky weather camera of TA is composed of a CCD camera, a fisheye-lens, a camera housing and an image server. The CCD camera is WAT120N+ (produced by Watec), and the fisheye-lens is FE185C057HA-1 (produced by Fijinon). The camera is housed in an aluminum case with a plastic dome-window. We used mm-Eye (produced by Brains Co.) as camera control and images server. Fig. \ shows image of this system. We have ordered all major parts with a generic product and realized a low cost night-sky monitor for less than $\$ 7,000$. This system is sensitive to visible light and has a wide field of view $\left(185^{\circ} 01^{\prime} \times 154^{\circ} 08^{\prime}\right)$ and enough pixels $(768$ $\times 494)$ to recognize individual stars. The brightness at each pixel is evaluated of 256 levels. Nightsky photography is taken at an exposure of 8 seconds per minute and is stored on a server installed at facilities such as FD stations.

\section{Analysis}

Examples of the night-sky images obtained by the system are shown in Fig. [3. These images show the weather change at night and it is easy to imagine that the clear sky is gradually covered with clouds. In the image of a clear night, we are able to find the Milky Way and many stars and have an overall higher brightness. On the other hand, cloudy sky images are dark overall because there are no stars. Partially cloudy weather suggests that clouds are present in areas where stars are not visible in the image. The edge of the night sky image shows near the ground and you can see the lights of the nightbering nightboring town scattered by aerosols. Night weather condition is estimated by the brightness of the night sky image, and we should consider difference of brightness between center and edge of the field of view. In addition, there are some noisy pixels that are always bright in the photo, and these noisy pixels are excluded our analysis.

We estimate the weather at night from these pictures using two strategies. One is the evaluation is the brightness of the night sky image, and the other is using the number of stars in the image.

Comparing the difference in brightness distribution between clear sky and cloudy sky images in Fig. 团 for the brightness evaluation. 
$02: 00$

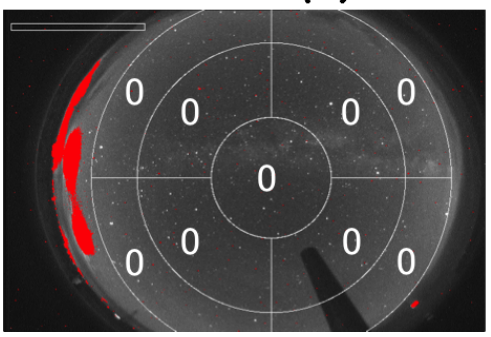

$06: 23$

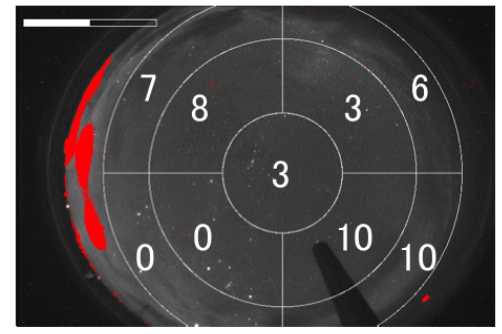

$11: 00 \quad(100)$

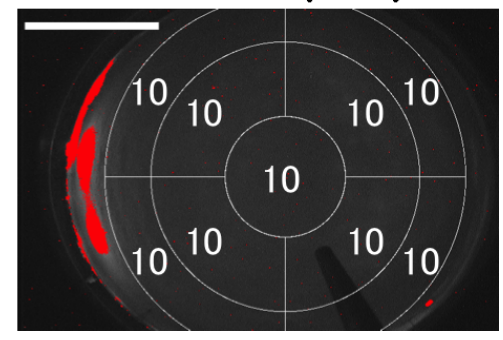

Figure 5: Example of the cloud score in 2014/Nov/20. The number of right side on the time is total cloud score.

The clear sky image has pixels with the maximum brightness value (255) in most areas, the brightness value of 160 or more was not obtained in all areas in the overcast image. The cloud evaluation values of each region is the value that obtained by subtracting 155 from the maximum brightness value in each region and dividing by 10 . In order to take into account the difference of brightness, we compared brightness of nine areas divided as Fig. [1. We named this evaluation "Cloud score". The Cloud score of the example images is also shown in Fig. [1.

Star detection searches for covers (clouds) in the field of view more directly. The number of stars over the night sky, called the "star count", is expected to be correlated with cloud cover. Cloud placement can be estimated by comparison with a star catalog. In the case of the star count method, the number of stars are counted by software in the same areas where cloud scores were calculated. In the star count method, we scan the pixels, and trace the outline of clusters of bright pixels when the brightness value exceeds the threshold by $3 \sigma$. A conceptual diagram of the star count analysis is shown in Fig.6. After the star detection routine, we remove the light sources other than stars. e.g. like an airplane by the size and the flatness of the cluster.

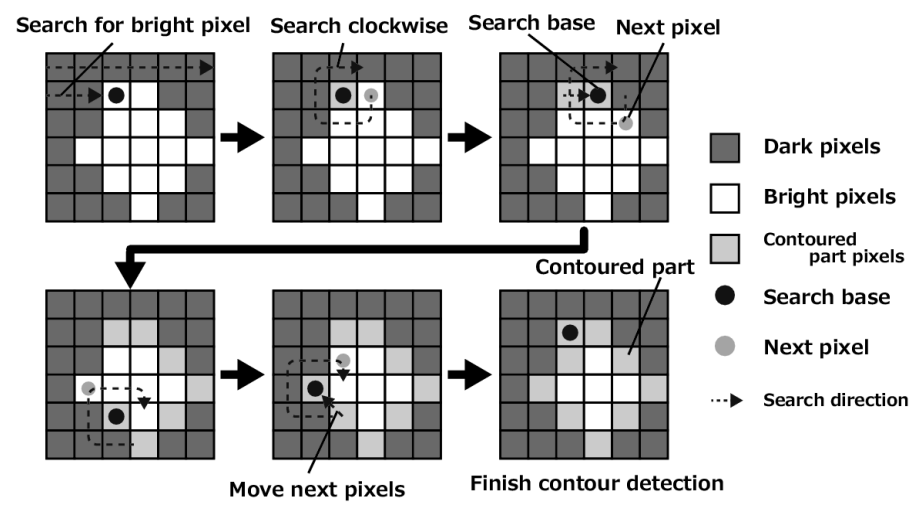

Figure 6: The method to identify stars in the sky image.

Comparison between the "cloud score" and "star count" and the Eye-scan code is shown in time in Fig. ㅁ. Black and dark gray solid lines show the cloud score and the number of stars, respectively. Gray triangles show the Eye-scan code. "cloud score" and "star count" as new anal- 
ysis methods of the night weather forecast indicate a good correlation. There is also a correlation between "cloud score" and the Eye-scan code, and our methods gives more detailed time variation.

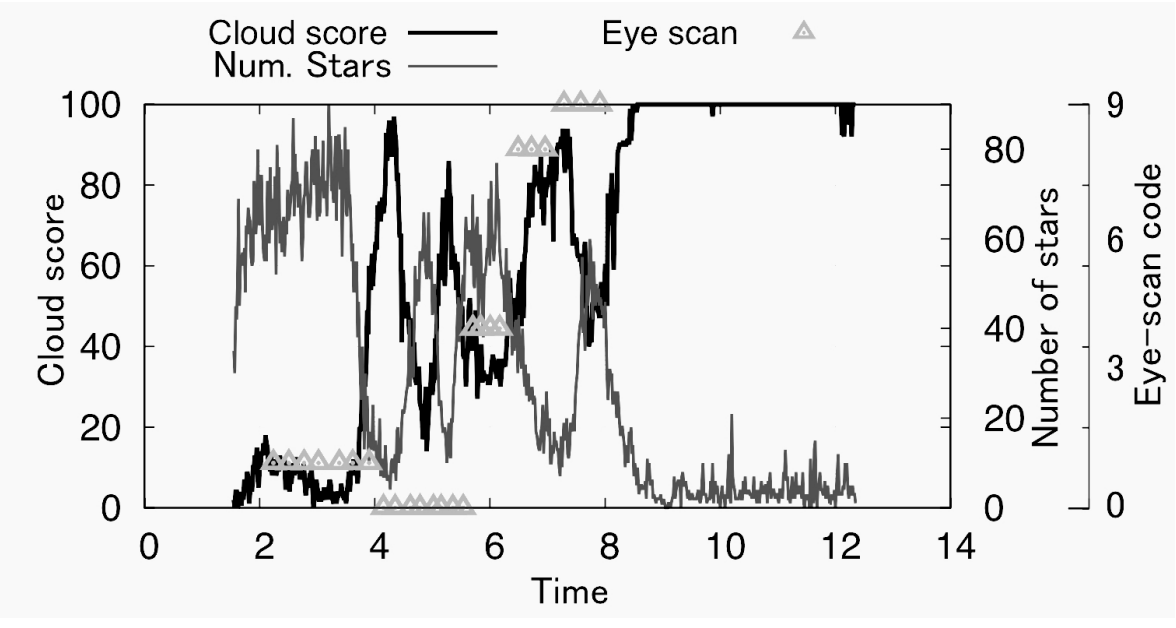

Figure 7: Comparison of 3 evaluations of the cloud in night sky on the time variation.

\section{Discussion}

The night sky monitor, created at low cost with an off the shelf CCD camera, is taking pictures of the stars and tells us of the existence of clouds. We focused on the brightness of the image and the number of stars and tried the nightly weather evaluation by two new methods. The outputs of these methods correlates with the night weather estimated by human-eyes in the existing method, and give us more detailed time variations. In other words, the two new analysis methods are effective, and it will be possible to use them for data selection.

On the other hand, we are also beginning to compare the stars catalog (the Smithsonian Astrophysical Observatory Star Catalog) with images of stars. The geometric adjustment of this technique is in the final stage as shown in the Fig. [8. By evaluating the magnitude and zenith angle of stars which can be detected and identified, it is expected to we can also see a correlation with atmospheric transparency etc..

\section{Acknowledgments}

The Telescope Array experiment is supported by the Japan Society for the Promotion of Science through Grants-in-Aids for Scientific Research on Specially Promoted Research (21000002) "BExtreme Phenomena in the Universe Explored by Highest Energy Cosmic Rays" and for Scientific Research (19104006), and the Inter-University Research Program of the Institute for Cosmic Ray Research; by the U.S. National Science Foundation awards PHY-0307098, PHY-0601915, PHY-0649681, PHY-0703893, PHY0758342, PHY-0848320, PHY-1069280, PHY-1069286, PHY-1404495 and PHY-1404502; by the National Research Foundation of Korea (2007-0093860, R32-10130, 2012R1A1A2008381, 2013004883); by the Russian Academy of Sciences, RFBR grants 11-0201528a and 13-02-01311a (INR), IISN project No. 4.4509 .10 and Belgian Science Policy under IUAP VII/37 (ULB). The foundations of Dr. Ezekiel R. and Edna Wattis Dumke, Willard L. Eccles and the George S. and Dolores Dore Eccles all helped with generous donations. The State of Utah supported the project through its Economic Development Board, and the University of Utah through the Office of the Vice President for Research. The experimental site became available through the cooperation of the Utah School and Institutional Trust Lands Administration (SITLA), U.S. Bureau of Land Management, and the U.S. Air Force. We also wish to thank the people and the officials of Millard County, Utah for their steadfast and warm support. We gratefully acknowledge the contributions 


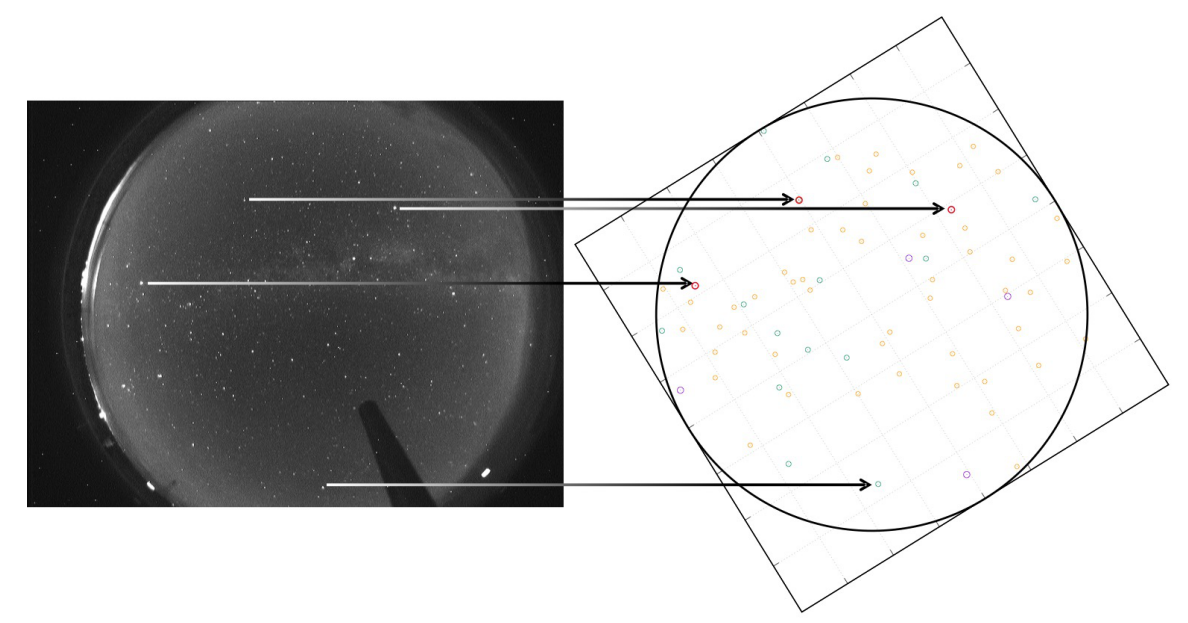

Figure 8: Comparison with star catalog.

from the technical staffs of our home institutions. An allocation of computer time from the Center for High Performance Computing at the University of Utah is gratefully acknowledged.

\section{References}

[1] H.Tokuno, et. al., NIM A, 676, 54-65 (2012) T.Abu-Zayyad et. al., NIM A, 689, 87-97 (2012). 